\title{
Pregnane $X$ receptor is associated with unfavorable survival and induces chemotherapeutic resistance by transcriptional activating multidrug resistance-related protein 3 in colorectal cancer
}

Yan Dong ${ }^{1}$, Zhe Wang ${ }^{1}$, Gan-feng Xie ${ }^{1}$, Chong Li ${ }^{1}$, Wen-wei Zuo ${ }^{1}$, Gang Meng ${ }^{2}$, Cheng-ping X ${ }^{2}$ and Jian-jun Li ${ }^{\text {* }}$

\begin{abstract}
Background: Although chemotherapy represents a predominant anti-cancer therapeutic modality, drug treatment efficacy is often limited due to the development of resistant tumor cells. The pregnane $X$ receptor (PXR) affects chemotherapeutic effects by regulating targets involved in drug metabolism and transportation, but the regulatory mechanism is poorly understood.

Methods: Oxaliplatin (L-OHP) content in tumor cells was analyzed by mass cytometry. The roles of PXR on cancer cell proliferation, apoptosis and tumor growth with L-OHP-treated were investigated by MTS, colony formation, flow cytometry and xenograft tumor assays. Luciferase reporter, Chromatin-immunoprecipitation and Site-directed mutagenesis were evaluated the mechanisms. The PXR and multidrug resistance-related protein 3 (MRP3) expressions were examined by western blot, RT-PCR or immunohistochemistry of TMA. Kaplan-Meier and Cox regression were adopted to analyze the prognostic value of PXR in colorectal cancer (CRC).

Results: PXR over-expression significantly increased oxaliplatin (L-OHP) transport capacity with a reduction of its content and repressed the effects of L-OHP on tumour cell proliferation and apoptosis. Conversely, PXR knockdown augments L-OHP-mediated cellular proliferation and apoptosis. Moreover, PXR significantly reduced the therapeutic effects of L-OHP on tumor growth in nude mice. Further studies indicated a positive correlation between PXR and MRP3 expression and this finding was confirmed in two independent cohorts. Significantly increased MRP3 expression was also found in PXR over-expressing cell lines. Mechanistically, PXR could directly bind to the MRP3 promoter, activating its transcription. The PXR binding sites were determined to be at -796 to $-782 \mathrm{bp}$ (CTGAAGCAGAGGGAA) and the key binding sites were the "AGGGA" (-787 to -783bp) on the MRP3 promoter. Accordingly, blockade of MRP3 diminishes the effects on drug resistance of PXR. In addition, PXR expression is significantly associated with poor overall survival and represents an unfavorable and independent factor for male or stage I + II CRC patient prognosis.

(Continued on next page)
\end{abstract}

\footnotetext{
* Correspondence: jianjunli@tmmu.edu.cn

'Department of Oncology, Southwest Hospital, Third Military Medical

University, No. 29, Gaotanyan Street, Shapingba District, Chongqing 400038,

People's Republic of China

Full list of author information is available at the end of the article
} 
(Continued from previous page)

Conclusions: PXR is a potential biomarker for predicting outcome and activates MRP3 transcription by directly binding to its promoter resulting in an increased L-OHP efflux capacity, and resistance to L-OHP or platinum drugs in CRC. Our work reveals a novel and unique mechanism of drug resistance in CRC.

Keywords: Colorectal cancer, Chemotherapy resistance, PXR, MRP3, Transcriptional regulation, Overall survival

\section{Background}

Colorectal cancer (CRC), a malignancy of the gastrointestinal tract, is the third most common tumor worldwide [1]. CRC patient outcomes have significantly improved due to the use of novel treatment modalities and complex treatment strategies, including optimized surgery, adjuvant chemotherapy and novel targeted biological agents. At an early stage, CRC is potentially curable by surgery followed by chemotherapy [2]. However, approximately $25 \%$ of CRC patients present with metastatic disease at diagnosis and are unlikely to undergo curative surgical resection, and many patients with metastatic disease will relapse after potentially curative resections $[3,4]$. For these patients, systemic chemotherapy is most often the treatment of choice for increasing survival and improving quality of life $[5,6]$. Thus, chemotherapy is one of the most important treatments for malignancies in humans [7]. Previous studies have revealed that the response rate after initial chemotherapy treatment is approximately $50 \%$ for CRC patients $[8,9]$. However, only $10 \%$ of CRC patients respond to secondary treatments [8]. These data suggest that there is a high degree of resistance to current chemotherapies.

The pregnane $\mathrm{X}$ receptor (PXR/NR1I2) is a ligandactivated transcription factor belonging to the nuclear hormone receptor (NR) superfamily [10]. PXR is expressed in many normal human tissues, including ovary, small intestine, colon, liver, breast, heart, and prostate [11]. Furthermore, increased PXR expression has been reported in various tumors, such as breast, ovarian, colon and endometrial cancers [12-17]. Further studies have shown that PXR plays an important role in the development of resistance to certain chemotherapeutic cancer treatments [18-22]. PXR activates many drug-metabolizing enzymes and drug transporters, resulting in altered drug clearance [23]. Our previous study showed that the mRNA expression of MRP3 significantly correlates with PXR expression in CRCs, suggesting that MRP3 might be involved in PXR-mediated chemotherapeutic resistance [16]. However, the specific role and precise molecular mechanism of PXR- and MRP3mediated chemotherapeutic resistance remain unclear.

In the present study, we demonstrated that PXR significantly reduced the amount of oxaliplatin (L-OHP) in tumor cells, and prevented L-OHP-mediated inhibition of cellular proliferation and induction of apoptosis. Our nude mouse model showed that PXR reduced the efficacy of L-OHP treatment on tumor growth. Further analysis revealed a positive correlation between MRP3 and PXR expression $(P=0.0023)$, which was confirmed in two independent cohorts from The Cancer Genome Atlas (TCGA) database. We also detected increased MRP3 expression in PXR over-expressing cell lines. Moreover, luciferase reporter, ChIP and site-directed mutagenesis assays demonstrated that PXR could directly bind to the MRP3 promoter, and that the key binding site were located at -796 to $-782 \mathrm{bp}$ on the MRP3 promoter. Further investigations showed that PXR expression was associated with poor prognosis and was an independent prognostic factor in CRC. Our data reveal that PXR can transcriptionally activate MRP3 expression by directly binding to its promoter, which increases drug efflux and results in CRC resistance to L-OHP or platinum drugs.

\section{Methods}

\section{Patient samples}

A total of 93 colorectal cancerous and 87 corresponding noncancerous tissues were obtained from the Southwest Hospital in Chongqing, China. This study was approved by the ethics committee of Southwest Hospital. Informed consent was obtained from all recruited patients.

\section{Tissue microarray generation and immunohistochemical analysis}

Noncancerous adjacent tissues were compared with normal tissue, stained with haematoxylin-eosin and reviewed by at least 2 pathologists. The tissue microarray (TMA) containing tissues from 93 tumors with 87 pairs of tumor and matched peritumoural tissues, was constructed (in collaboration with Shanghai Biochip Company Ltd, Shanghai, China) as previously described [24].

Immunohistochemistry (IHC) was performed using PXR and MRP3 antibodies (both 1:200; both from Santa Cruz Biotechnology; sc-48403 and sc-5774). Tumor cell staining was considered positive when immunoreactivity was greater than or equal to $10 \%$. Positive staining was divided into 5 categories, and staining intensity was graded according to 4 levels as previously described [25]. The expression levels were defined by the sum of positive staining and intensity. 
The construction and transfection of expression and siRNA vectors for PXR

Expression and siRNA vectors for PXR were constructed and transfected as previously described [26]. The stably PXR-transfected cells were selected using G418 (Calbiochem, La Jolla, CA, USA). Cell clones were obtained using the cylinder method.

\section{RT-PCR and western blotting (WB) analysis}

The mRNA expression analysis was performed using RT-PCR. Series PCR assays with different cycle numbers were performed to determine the linear phase of amplification. Based on the pilot experiments, the appropriate cycles were chosen. The primers are listed in Additional file 1: Table S1.

Sixty micrograms of protein was resolved by $10-15 \%$ sodium dodecyl sulphate- polyacrylamide gel electrophoresis and transferred onto polyvinylidene difluoride membranes (Millipore Corporation, Bedford, MA, USA). After blocking, the membranes were incubated with primary antibodies overnight at $4{ }^{\circ} \mathrm{C}$. The proteins were detected by chemiluminescence (Pierce, Rockford, IL, USA) after incubation with secondary antibodies. The probed membranes were stripped and incubated with $\beta$ actin monoclonal antibody (1:1200; Sigma). The primary antibodies used were PXR mouse monoclonal antibody (1:1000, Santa Cruz Biotechnology, sc-48403) and MRP3 goat polyclonal antibody (1:800; Santa Cruz Biotechnology, sc-5774). The secondary antibodies used were horseradish peroxidase-conjugated anti-mouse and antigoat antibodies (both 1:2000, Jackson ImmunoResearch Laboratories, Inc., West Grove, PA, USA).

\section{Oxaliplatin drug treatment}

For L-OHP content measurement, tumor cells were treated for $2 \mathrm{~h}$ at a concentration of $20 \mathrm{mmol} / \mathrm{L} \mathrm{L}-\mathrm{OHP}$ (Hengrui Medicine, Lianyungang, Jiangsu Province, China); For the functional experiments in vitro, tumor cells were exposed for $24 \mathrm{~h}$ to $20 \mathrm{mmol} / \mathrm{L} \mathrm{L}-\mathrm{OHP}$. The culture medium was replaced on a daily. For the tumor formation in vivo, the nude mice were injected once every 3 days with $5 \mathrm{mg}$ of L-OHP (dissolved in normal saline) $/ \mathrm{kg}$ body weight. Controls were treated with the same volume of normal saline.

\section{Measurement of L-OHP content in tumour cells by mass cytometry}

The transfected, L-OHP-treated tumor cells were analyzed using a CyTOF1 mass cytometer (DVS Sciences, Richmond Hill, Ontario, Canada). The instrument settings and initial post-processing parameters were set as previously described [27-29]. Cells were measured at approximately 600 cells per second. Noise reduction was activated, and cell extraction parameters were as follows: cell length range was set from 15- to- 65 pushes, and the lower convolution threshold was set to 15 .

\section{Colony-formation and MTS assay}

HCT116 and LOVO cells were plated in 12-well plates at $3 \times 10^{5}$ cells per well. After culturing for $24 \mathrm{~h}$, the cells were transfected with PXR or vector plasmids. After $48 \mathrm{~h}$ of transfection, the cells were collected, diluted 1:5, plated in 12-well plates and selected with $0.8 \mathrm{mg} / \mathrm{ml}$ of G418 for 14 days to establish stable clones and for subsequent treatment with L-OHP. Surviving colonies were stained by using Giemsa's azur eosin methylene blue solution (Merck, Darmstadt, Germany) and counted. These experiments were performed in triplicate.

HCT116 and LOVO cells were plated in 96-well plates at $3.5 \times 10^{3}$ cells per well, transfected with PXR or empty vector plasmids, and treated with L-OHP. For knockdown, SW480 cells were plated in 96-well plates at $3 \times$ $10^{3}$ cells per well, transfected with PXR siRNA or negative control plasmids, and treated with L-OHP. Cell proliferation was evaluated using the Cell Proliferation Reagent MTS (CellTiter $96^{\circ}$ Aqueous One Solution Cell Proliferation Assay, Promega) on days 1, 2, and 3. The assay was carried out in triplicate in three independent experiments.

\section{Flow cytometry assay}

PXR or empty vector-transfected cells $\left(3.5 \times 10^{5}\right.$ cells per well) were harvested at $48 \mathrm{~h}$ post-transfection and LOHP treatment, and fixed in $70 \%$ ethanol overnight at 4 ${ }^{\circ} \mathrm{C}$. The cells were stained with propidium iodide (BD Pharmingen, San Jose, CA, USA). Fifty thousand cells were sorted by FACSCalibur System (BD Biosciences, Franklin Lakes, NJ, USA), and cell cycle profiles were analysed using the ModFit software (Verity Software House, Topsham, ME, USA). Apoptosis was also assessed by Annexin V-APC/7-amino-actinomycin D staining (KeyGEN, Nanjing, China). Briefly, cells were harvested, washed with phosphate-buffered saline, resuspended in $500 \mu \mathrm{l}$ of binding buffer, mixed with $5 \mu \mathrm{l}$ of Annexin V-APC and $5 \mu$ of 7-ADD, incubated for 5-15 min in the dark. Fifty thousand cells were analyzed using a FACSCalibur cytometer. The Annexin V-positive cells were considered apoptotic cells and analysed using the ModFit software. The assays were carried out in triplicate in three experiments.

\section{In vivo tumourigenicity}

To assess the effects of PXR expression and L-OHP treatment on tumour formation in vivo, the PXR- or empty vector-expression stable HCT116 cells were injected subcutaneously into the right flanks of 6-weekold male Balb/c nude mice that were subsequently subjected to L-OHP treatment. Tumor volume was 
calculated as $0.5236 \mathrm{~L} 1$ (L2) ${ }^{2}$ [30]. The developing tumors were observed during the following 5 weeks, after which the mice were sacrificed. All mouse experiments were approved by the Institutional Animal Care and Use Committee of Third Military Medical University, China.

\section{Site-directed mutagenesis of binding site assay}

The site-directed mutagenesis assays were performed as previous study (26). The binding sites in MRP3 promoter constructs were mutated or deleted using a QuikChange Lightning Multi Site-Directed Mutagenesis Kit (Stratagene, La Jolla, CA, USA) according to the instructions, and the mutations or deletions were confirmed by direct DNA sequencing.

\section{Luciferase reporter and chromatin-immunoprecipitation} (ChIP) assay

Cells were seeded in 24-well plates at $3.5 \times 10^{4}$ cells per well. After an overnight incubation, the cells were transfected with a DNA mixture including pGL3-MRP3 promoter-luciferase, pIRES2-EGFP-PXR or vector, as well as the internal pRL-TK plasmids. Luciferase activities were measured at $36 \mathrm{~h}$ post-transfection using a dual-luciferase reporter kit (Promega). Each experiment was performed in triplicate and repeated three times.

Chromatin-immunoprecipitation (ChIP) analysis was performed using a ChIP Assay Kit (Cell Signaling Technology) according to the manufacturer's protocols. The immunoprecipitated and input DNA were used as templates for RT-PCR analysis, and the primers are listed in Additional file 1: Table S1.
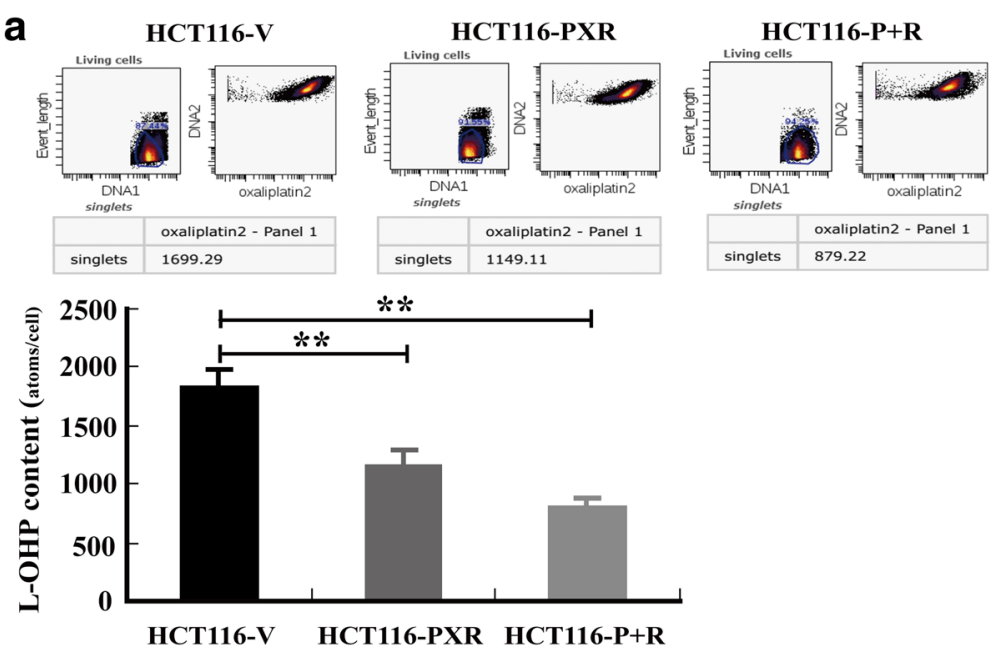

b
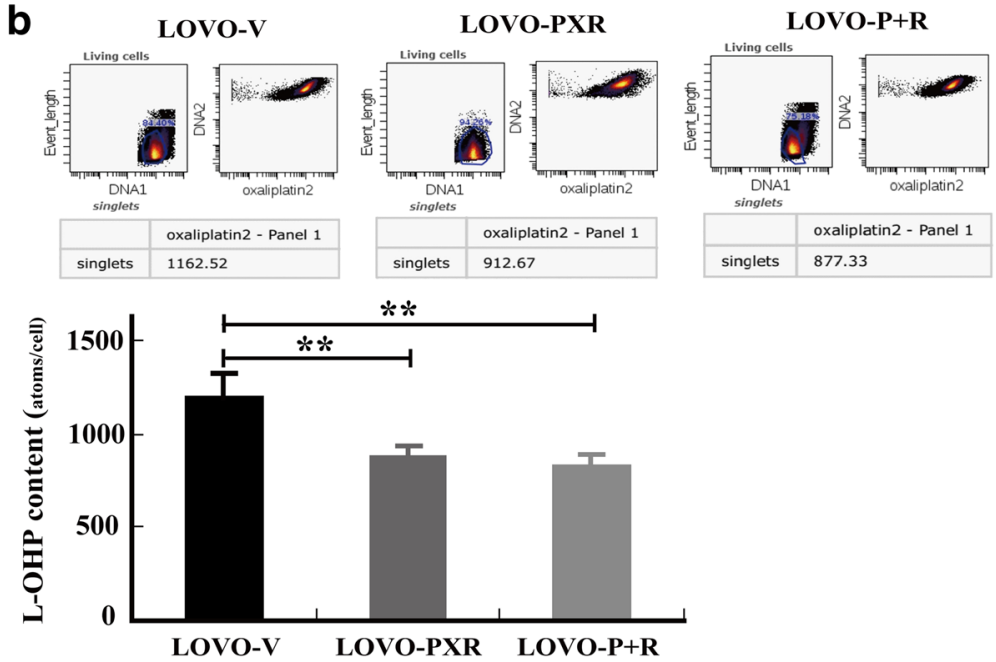

Fig. 1 PXR over-expression decreases L-OHP levels in tumor cells. The transport and uptake of L-OHP in tumor cells were assessed by mass cytometry. L-OHP content in PXR or PXR + RXRA stably transfected tumor cells was reduced compared with that in empty vector stably transfected tumor cells on average in both HCT116 (a) and LOVO (b) cells. Tumour cells were treated for $2 \mathrm{~h}$ with $20 \mathrm{mmol} / \mathrm{L}$ oxaliplatin. P + R, PXR + RXRA. The unit shown is Pt atoms/cell 


\section{Statistical analyses}

Statistical analyses were performed using SPSS 13.0 software (SPSS, Inc., Chicago, IL, USA). The results are expressed as the mean \pm standard deviation (s.d.). The data were analysed by Student's $t$ and chi-square (2-sided) tests. Patient clinical and pathological characteristics were compared by Pearson $\chi^{2}$ test. Overall survival (OS) was calculated according to Kaplan-Meier and Cox regression. The $\mathrm{p}$ values less than 0.05 were considered significant.

\section{Results}

\section{PXR decreases oxaliplatin (L-OHP) levels in tumor cells}

To determine the role of PXR in L-OHP-treated tumor cells, L-OHP transport and uptake were assessed by mass cytometry in tumor cells transfected with PXR or empty vector, as well as in those transfected with PXR + RXRA, which generally forms a heterodimer that transcriptionally activates target genes. Our results revealed that the L-OHP content in tumor cells stably transfected with PXR or PXR + RXRA was on average significantly lower than that in tumor cells transfected with empty vector in both HCT116 and LOVO cells (Fig. 1a, b). These results showed that PXR notably increased the L-OHP efflux capacity of tumor cells, thereby reducing intracellular L-OHP content.

\section{PXR prevents L-OHP-mediated inhibition of cancer cell proliferation and apoptosis}

To explore the functional roles of reduced L-OHP content, we performed cell proliferation assays in L-OHPtreated PXR over-expressing cells. The MTS data and colony-formation assays indicated that PXR prevented the L-OHP-mediated suppression of tumor cell proliferation (Fig. 2a-c, Additional file 1: Table S2). To exclude the possible direct role of PXR over-expression, the data

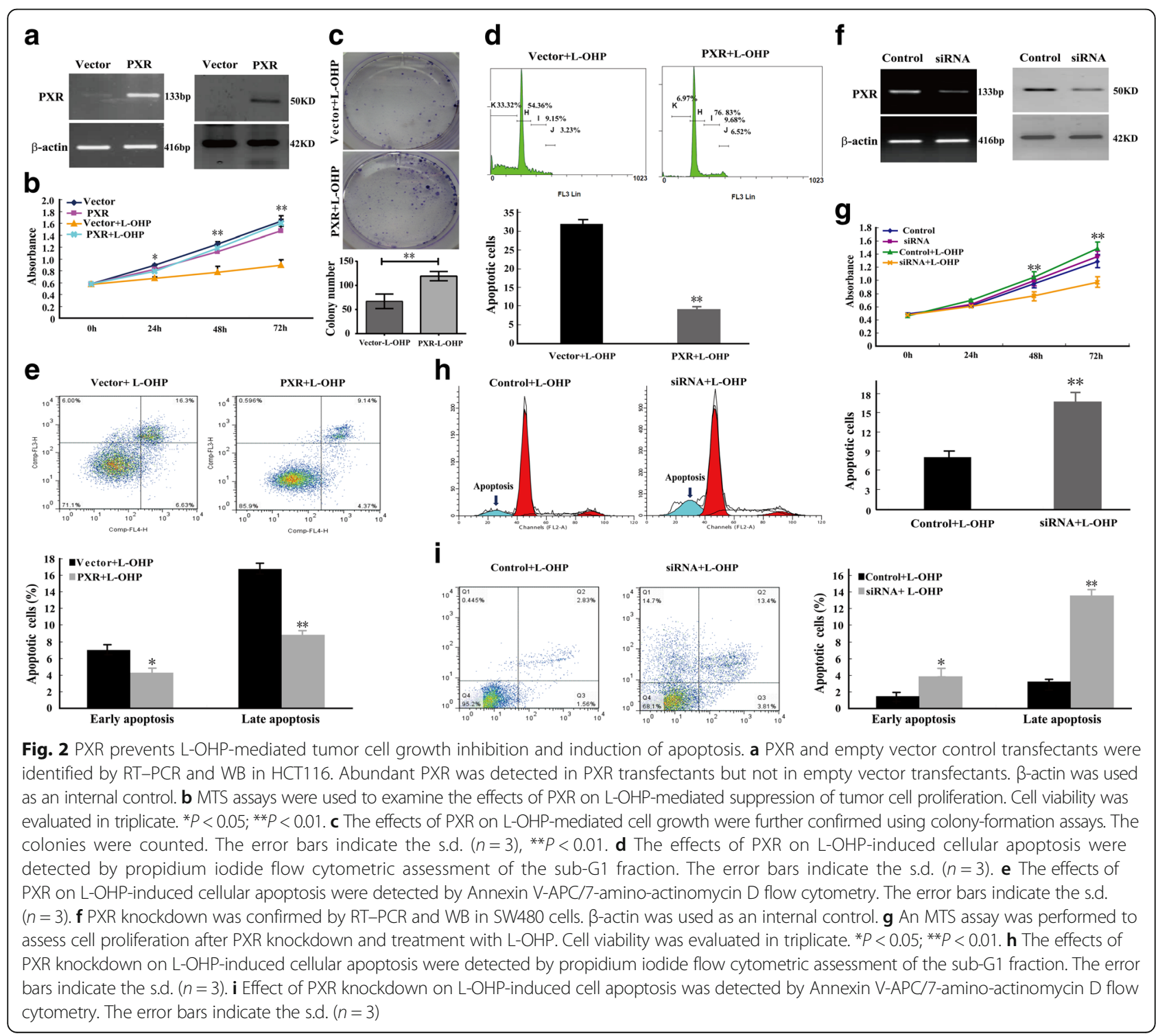


of MTS assay presented also include groups without LOHP treatment (Fig. 2b). To further examine the effects of PXR over-expression on the L-OHP-mediated inhibition of cellular proliferation, we measured the percentage of sub-G1 phase cells and also performed Annexin V-APC/7-amino-actinomycin D staining followed by flow cytometric analysis. The PXR-transfected tumor cells exhibited a significantly reduced percentage of subG1/apoptotic cells as compared with empty vectortransfected cells that were treated with L-OHP (Fig. 2d). The double staining analysis revealed that PXR overexpression decreased the percentage of early- and latestage apoptotic cells after L-OHP treatment (Fig. 2e). These results demonstrated that PXR over-expression promoted tumor cell proliferation and inhibited tumor cell apoptosis during L-OHP treatment.

To further demonstrate that PXR expression can influence tumor cell proliferation and apoptosis during LOHP treatment, we constructed a PXR-knockdown SW480 cell model (Fig. 2f). Cell proliferation and flow cytometry assays revealed that PXR knockdown inhibited tumor cell proliferation and promoted apoptosis after L-OHP treatment (Fig. 2g-i). The data of MTS assay presented also include groups without L-OHP treatment (Fig. 2g). These results further demonstrated that PXR promoted tumor cell proliferation and inhibited L-OHP-induced tumor cell apoptosis.

\section{PXR reduces the curative effect of L-OHP on tumor growth in nude mice}

Our experiments demonstrated that PXR can promote tumor cell proliferation and inhibit tumor cell apoptosis during L-OHP treatment in vitro. To test this conclusion in vivo, we assessed tumourigenicity in a nude mouse model of stable PXR-expressing cells and L-OHP treatment. Tumor volumes were significantly larger in L-OHPtreated mice injected with PXR-transfected cells compared with those injected with empty vector-transfected cells (Fig. 3a, b). PXR over-expression resulted in an increase of the mean weight of tumors collected at 5 weeks after cell inoculation (Fig. 3c, d). To exclude the possible direct role of PXR, the data of tumor volumes and weights from nude mice were also presented without L-OHP treatment (Fig. 3e). Collectively, these data indicated that PXR significantly reduced the curative effects of L-OHP on tumor growth. a

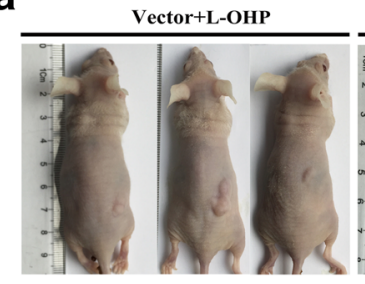

b

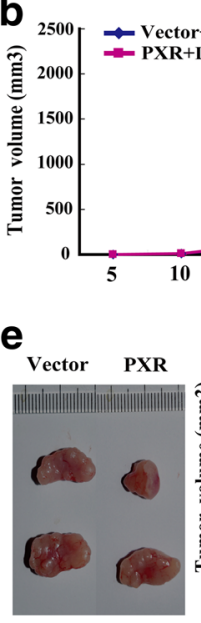

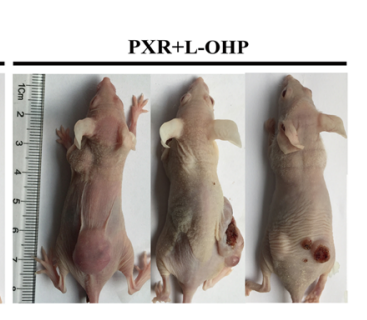

\section{c}

d
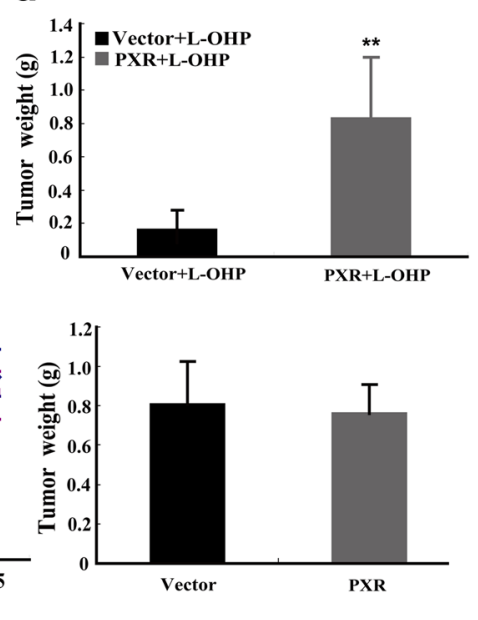

Fig. 3 PXR reduces the curative effect of L-OHP on tumor growth in nude mice. $\mathbf{a}$, $\mathbf{b}$ The tumor growth curve of PXR-expressing cells was compared with vector-expressing cells after L-OHP treatment. Tumor growth was assessed in nude mice that were subcutaneously injected in the right flank with $5.0 \times 10^{6}$ stable transfectants. Points represent the mean tumor volumes of three independent experiments $(n=3)$. $\mathbf{c}$, $\mathbf{d}$ Tumor weights from the PXR and vector groups were measured. The results were obtained from three independent experiments. ${ }^{*} P<0.05 ;{ }^{*} P<0.01$. The error bars indicate the s.d. $(n=3)$. e The tumor growth curve and tumor weights of PXR-expressing cells were compared with vector-expressing cells. The results were obtained from three independent experiments. The error bars indicate the s.d. $(n=3)$ 
PXR positively correlates with MRP3 expression in CRC To investigate the protein levels of PXR and MRP3 expression in human CRCs, we performed IHC for PXR and MRP3 in a TMA containing 93 cancer and 87 paracarcinoma colorectal tissues. PXR protein levels were increased in most cancer tissues compared with paracarcinoma tissues, and the mean expression of PXR in cancer tissues (8.366) was significantly increased compared with para-carcinoma tissues $(3.586) \quad(P<0.001)$ (Fig. 4a, b). MRP3 protein levels were also increased in most cancers compared with para-carcinoma tissues, and the mean expression of MRP3 in the cancer tissues (6.361) was significantly increased compared with that of the para-carcinoma tissues (4.083) $(P<0.001)$ (Fig. 4a, b). These results suggested that PXR and MRP3 were frequently over-expressed in tumor tissues compared with in adjacent tissues.

To further evaluate whether PXR correlates with MRP3 expression, we assessed the relationship between MRP3 and PXR protein expression. Statistical analysis revealed a significant positive correlation between PXR and MRP3 protein expression in CRC (Fig. 4c, $P=$ $0.0023)$, a result that was further confirmed in two independent cohorts from TCGA database at mRNA level (Fig. 4d). These results indicated that PXR might increase platinum drug metabolism, causing resistance to platinum drugs by up-regulating MRP3 gene expression.

\section{PXR activates MRP3 transcription by directly binding to its promoter}

To elucidate the molecular mechanism underlying the PXR-mediated regulation of MRP3, gene expression profiling was performed using stable transfectants. Our data revealed that MRP3 expression increased with the increase of PXR expression (Fig. 5a). As PXR is a transcription factor, we first analyzed whether it could directly regulate MRP3 at the transcriptional level. To test this hypothesis, we performed a luciferase reporter assay, which revealed that PXR over-expression significantly enhanced the activity of the MRP3 promoter $(-2052$ to $+117 \mathrm{bp})$, suggesting that PXR regulates MRP3

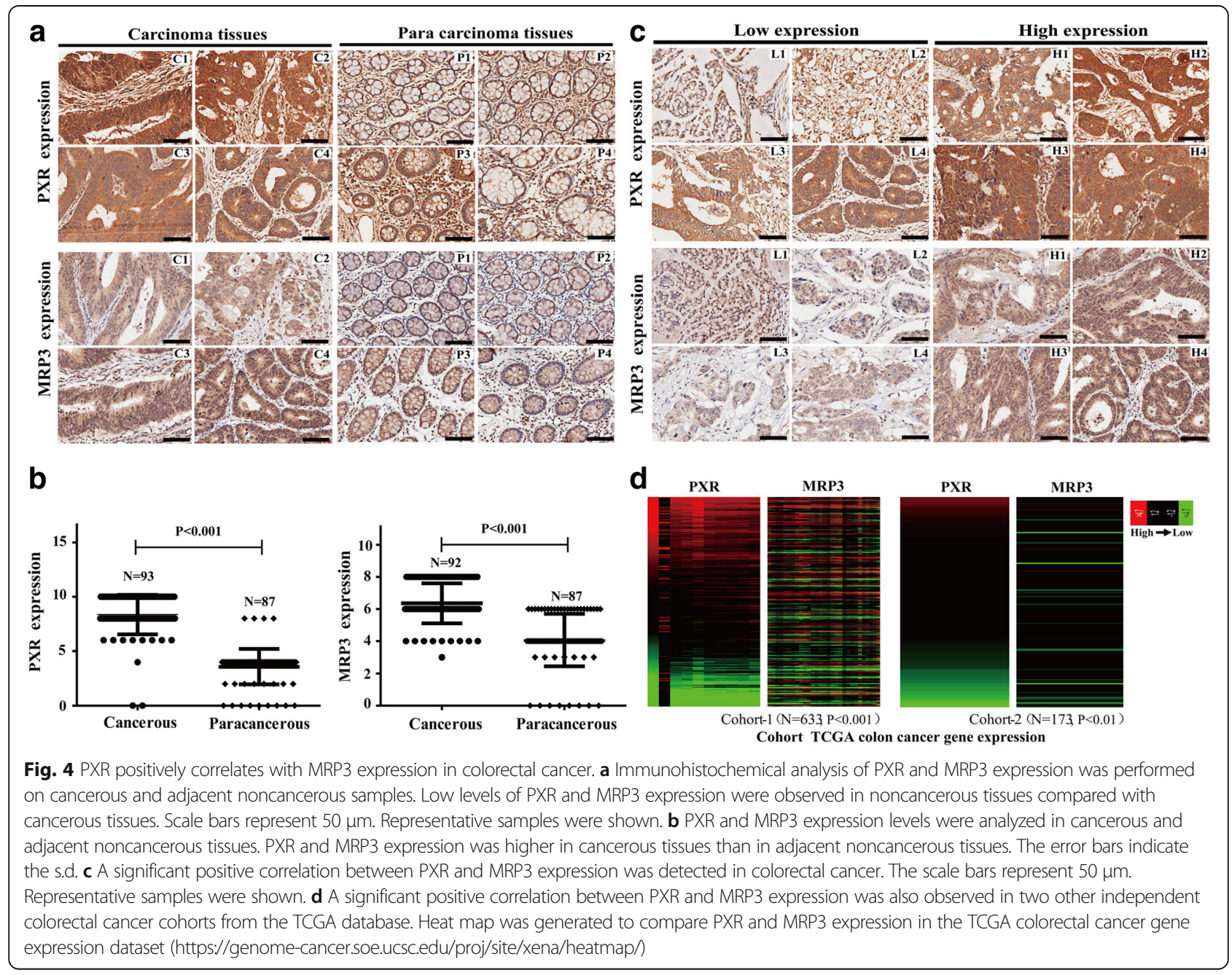




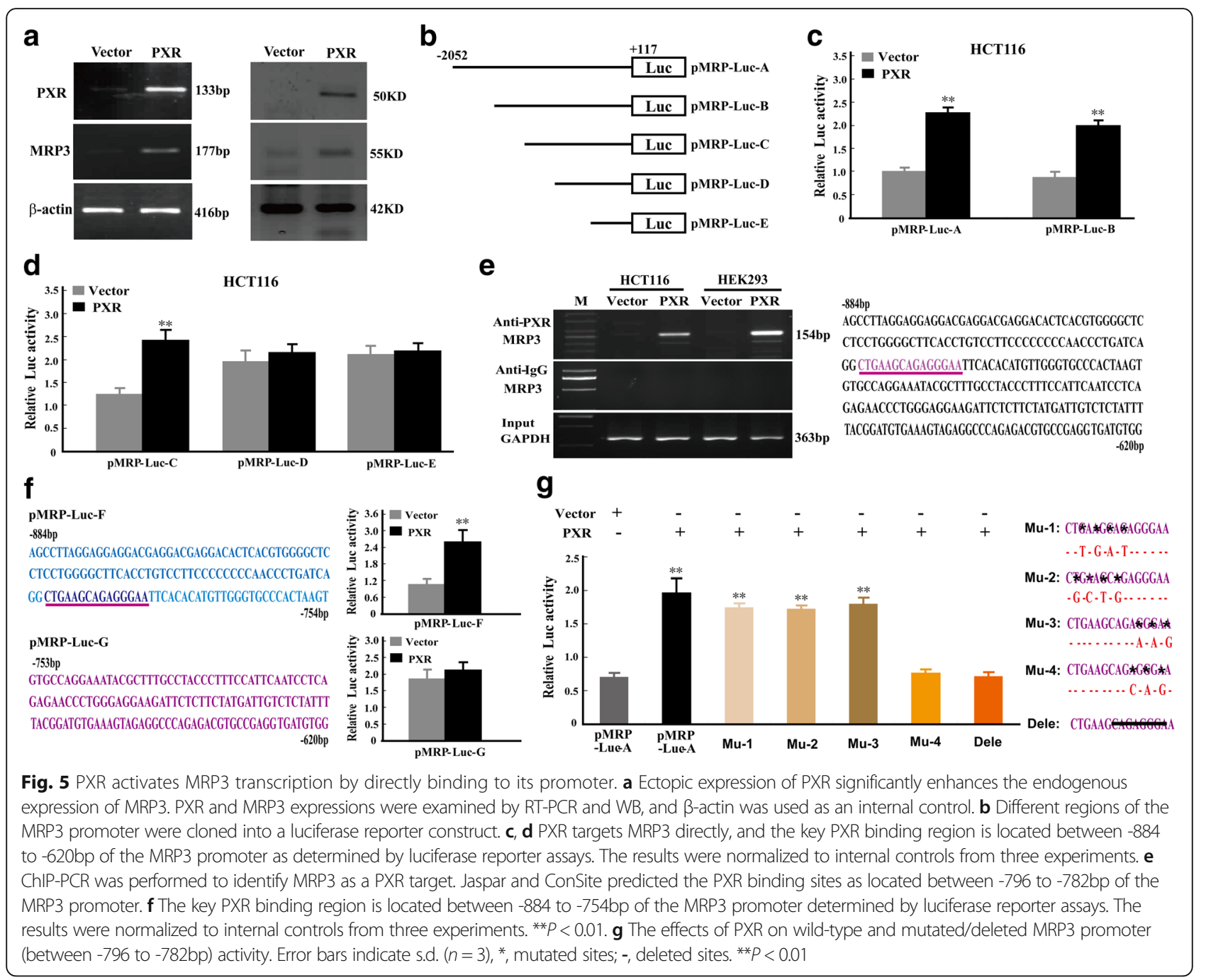

by directly binding to its promoter. To identify the PXRbinding site(s) within the MRP3 promoter, different regions of the MRP3 promoter (pMRP-Luc-B- $\sim$ E) were analyzed using luciferase reporter assays. Our results showed that the key region required for PXR binding was located between -884 to $-620 \mathrm{bp}$ of the MRP3 promoter (Fig. 5b-d). This result was confirmed by a ChIPPCR assay, and aided by the binding profile database of Jaspar and ConSite, we determined that the crucial binding region resides within -796 to $-782 \mathrm{bp}$ (CTG AAGCAGAGGGAA) of the MRP3 promoter (Fig. 5e). To confirm the binding sites in the "CTGAAGCAGAGGGAA" region of the MRP3 promoter, two different regions of MRP3 promoter between -884 and -620bp (pMRP-Luc-F and G) were then analyzed using luciferase reporter assays. The data revealed that the region containing "CTGAAGCAGAGGGAA" region was required for PXR binding (Fig. 5f). To further investigate the key binding sites, constructs with site-directed mutagenesis of binding sites were generated. When the
PXR-binding sites, AGGGA- $-(-787$ to -783bp), in MRP3 promoter were mutated or deleted, the activation was lost (Fig. $5 \mathrm{~g}$ ). These findings demonstrated that MRP3 was a direct PXR target gene in CRC.

\section{MRP3 is a key player in PXR mediated drug resistance}

To define whether MRP3 was required for PXR mediated drug resistance in CRC, we performed a rescue experiment by knocking down MRP3 in PXR overexpressed cells followed by testing cellular proliferation, apoptosis and intracellular L-OHP content. The results of rescue experiment demonstrated that blockade of MRP3 by small interfering RNA (siRNA) significantly diminished the effect of PXR over-expression on cancer cell proliferation, apoptosis and intracellular L-OHP content (Fig. 6a-d). These data indicate that the contribution of MRP3 is very important for PXR mediated drug resistance in CRC. 
a

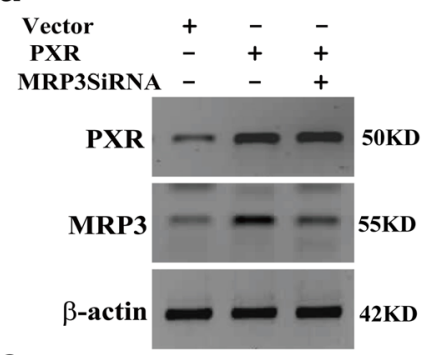

b

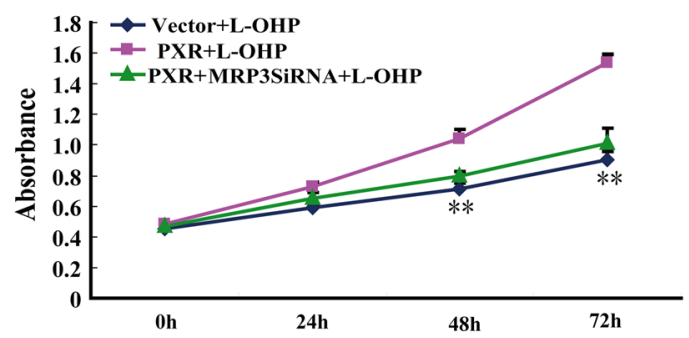

PXR+MRP3SiRNA+L-OHP

C
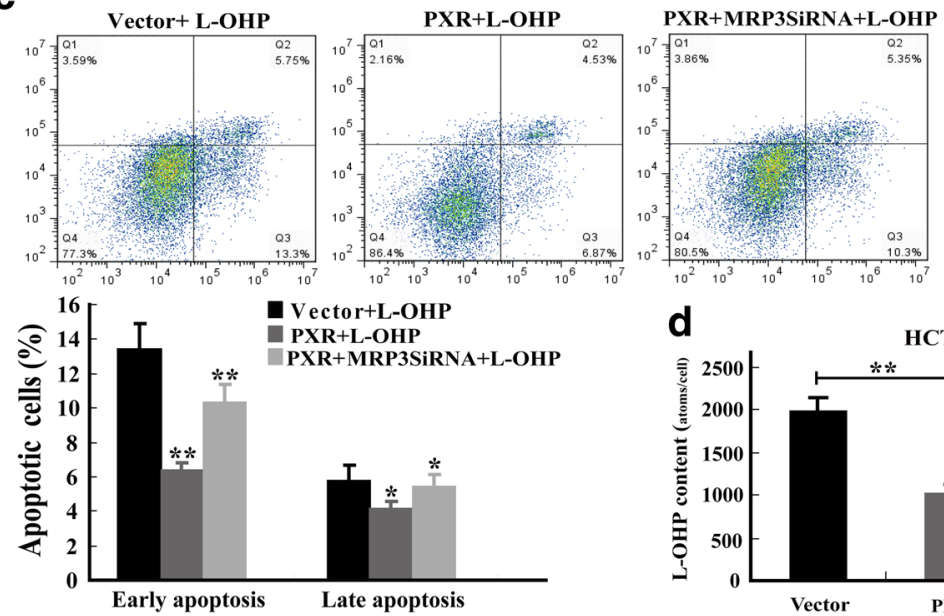

d

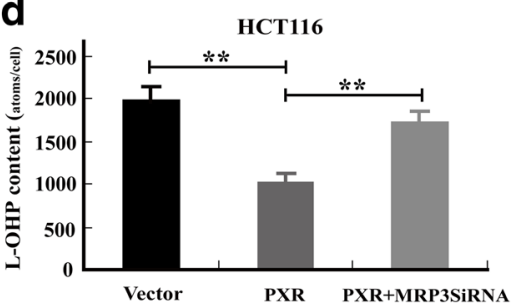

Fig. 6 MRP3 is a functionally target gene of PXR in drug resistance. a The expression of PXR and MRP3 was analyzed by WB at 48h after transfecting with MRP3 small interfering RNA (siRNA). The MRP3 siRNA was purchased from Santa Cruz Biotechnology. $\mathbf{b}$ Analysis of the effects of L-OHP on cancer cell proliferation when different PXR and MRP3 expression. Cell numbers were counted every day after treatment for 4 days. $\mathbf{c}$ Analysis of the effects of L-OHP on cancer cell apoptosis when different PXR and MRP3 expression. $\mathbf{d}$ The transport and uptake of L-OHP in tumour cells with different PXR and MRP3 expression were assessed by mass cytometry

PXR expression is significantly associated with poor overall survival in $<\mathrm{h} 2>\mathrm{CRC}$ patients

To determine the importance of PXR in CRC, the clinical and prognostic significance of PXR expression were analyzed. Based on the quantified positive staining of tumor cells, PXR expression was classified into two groups around the median score: as high (>median) and low ( $\leq$ median). After investigating the association between PXR expression and the clinicopathological features of CRC patients, PXR expression was significantly correlated with patient age $(P=0.011)$, but not with sex, lymph node status, histological grade, tumor size and clinical stage (Additional file 1: Table S3 and Additional file 2).

To investigate the correlation between PXR expression and survival of CRC patients, we next examined the contribution of PXR expression to patient OS. Kaplan-Meier survival analysis revealed a poorer OS in cancer patients characterized with high PXR expression compared with patients with low PXR expression $(P=0.013$; Fig. 7a). To avoid bias, PXR expression and other parameters were examined in a multivariate Cox-regression analysis. PXR expression was found to be an independent prognostic factor [hazard ratio $(\mathrm{HR})=1.463,95 \%$ CI 1.049 2.041, $P=0.025]$, in addition to age $(\mathrm{HR}=1.040,95 \% \mathrm{CI}$ 1.000-1.081, $P=0.049$ ), for patient OS (Fig. 7a, Additional file 1: Table S4 and Additional file 2).

To determine the importance of other clinicopathological features on the correlation between PXR expression and OS of cancer patients, we stratified patients by PXR expression and other clinicopathological features followed by analysis of survival data. The results revealed that PXR expression was significantly associated with OS of male patients (univariate, $p=0.044 / \mathrm{multi}$ variate, $p=0.006$ ) or the patients at clinical stage I + II (univariate, $p=0.009 /$ multivariate, $p=0.014$ ) (Fig. 7b, c, Additional file 1: Table S5, Table S6 and Additional file 2). However, PXR expression was not statistically correlated with OS of female patients $(p=0.134)$ or the patients at clinical stage III + IV $(p=0.247)$. There are no different impacts about histological grade on the correlation between PXR expression and OS. These findings suggested that PXR was an unfavorable and independent prognostic factor for male or clinical stage I + II CRC patients. 

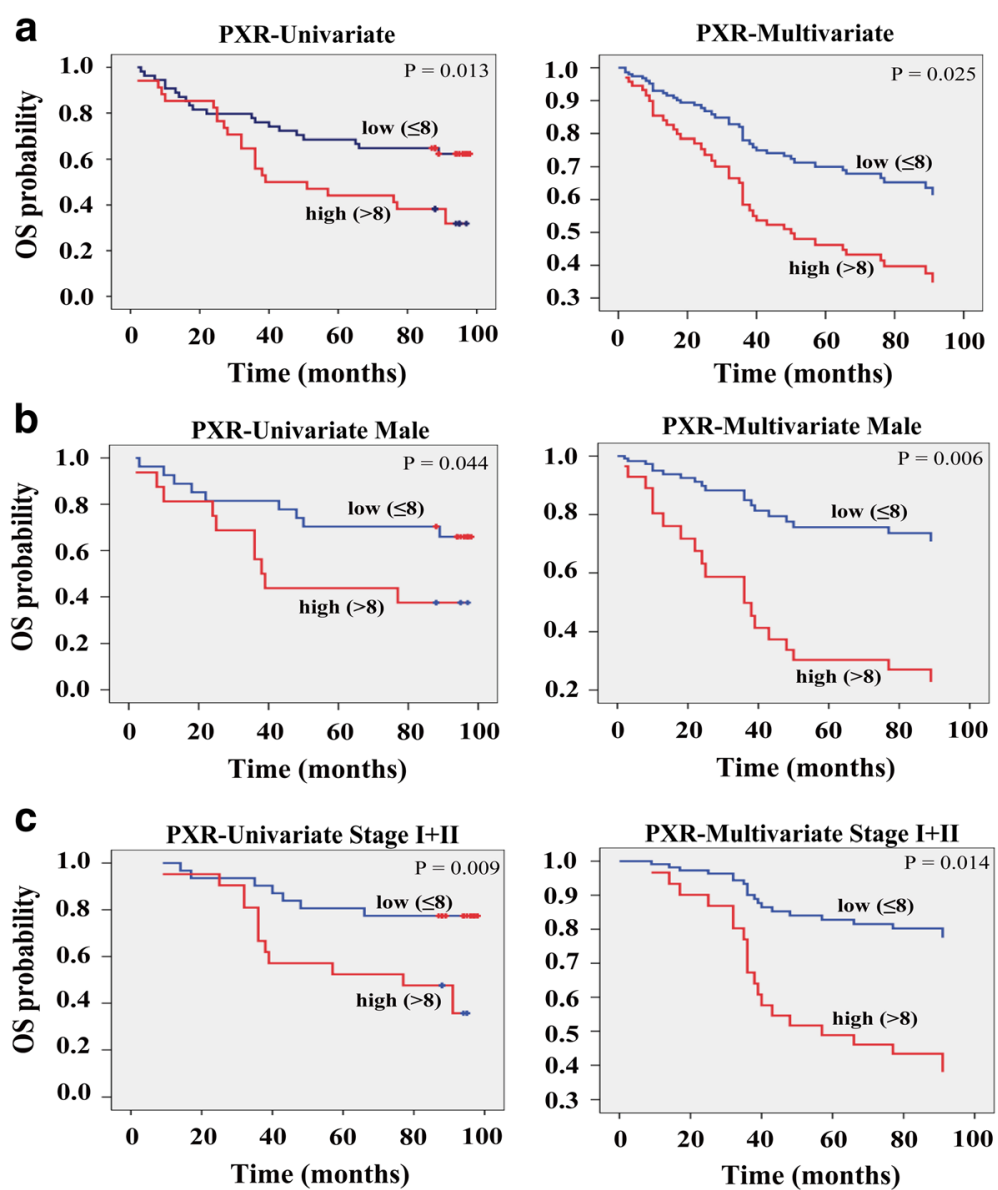

Fig. 7 PXR expression is significantly associated with poor overall survival in colorectal cancer patients. a Kaplan-Meier (univariate) and Cox regression (multivariate) survival analysis of PXR expression in 93 colorectal cancer patients. PXR expression is associated with poor OS and an independent prognostic factor in colorectal cancer patients. Low, staining weak $(\leq 8)$; High, staining strong $(>8)$. b Kaplan-Meier and Cox regression survival analysis of PXR expression in 47 male colorectal cancer patients. c Kaplan-Meier and Cox regression survival analysis of PXR expression in 56 stage I+ II colorectal cancer patients

\section{Discussion}

In this study, we investigated the molecular mechanisms of PXR-mediated MRP3 over-expression, which we demonstrate for the first time contributes to chemotherapeutic resistance in CRC. Our data demonstrated that PXR increased the L-OHP efflux capacity, reducing drug concentrations in tumor cells. Functional analysis revealed that PXR blunted L-OHP-mediated inhibition of cellular proliferation and inducing of apoptosis. Our nude mouse experiments showed that PXR reduced the efficacy of L-OHP treatment on tumor growth. TAM analysis revealed increased expression of PXR and MRP3 in cancer tissues and a positive correlation between MRP3 and PXR expression $(P=0.0023)$, which was further confirmed in two independent cohorts from the TCGA database. To investigate the mechanism of PXR-induced L-OHP resistance, MRP3 expression was assessed in cell models that differentially expressed PXR. The results revealed increased MRP3 expression in PXR over-expressing cells. Luciferase reporter and ChIP assays indicated that PXR can bind to the promoter of MRP3 to activate transcription and furthermore, that the PXR-binding sites are located at between -796 to $-782 \mathrm{bp}$ of the MRP3 promoter. Moreover, we found that PXR expression is an independent predictor for poor prognosis in CRC patients. These results suggest that PXR can transcriptionally activate MRP3 by directly binding to its promoter, increasing drug efflux capacity and resulting in resistance to platinum drugs.

Chemotherapy is the treatment of choice in patients with advanced or metastatic CRC. Chemotherapeutic resistance of tumor cells is crucial problem and a complicated process that involves multiple genes and steps. PXR is a member of the ligand-activated transcription factor superfamily, and its downstream target genes are involved in drug metabolism and transportation [20, 31]. Increasing evidence suggests that high PXR expression is associated with decreased treatment efficacy and increased chemoresistance against drug-based cancer treatments, including 
irinotecan, tamoxifen, paclitaxel, doxorubicin and vinblastine [32-39]. In this study, PXR increased the drug efflux capacity and reduced the amount of drug in tumor cells, attenuating the efficacy of L-OHP. PXR expression significantly decreased L-OHP.-mediated tumour cell growth inhibition and inducing of apoptosis. In our nude mouse experiments, PXR decreased L-OHP-mediated suppression of tumor growth. These results demonstrate that PXR enhances the resistance of CRC cells to the chemotherapeutic agent L-OHP.

Previous studies have indicated that PXR regulates a number of genes associated with drug resistance, such as cytochrome $\mathrm{P} 450$, multidrug resistance 1 and multidrug resistance-associated protein 2 [20, 31, 40]. Mounting evidence has shown that PXR is a master regulator of chemotherapeutic drug resistance in cancer treatment. However, the specific mechanism underlying this resistance remains unclear. MRP3 (also called ABCC3) belongs to the $\mathrm{ABCC}$ subfamily, which consists of 13 members in mammals and has been divided into the following 3 classes: the multi-drug resistance proteins (MRPs), the sulfonylurea receptors and the cystic fibrosis transmembrane conductance regulator (CFTR/ABCC7). MRP3 has not been extensively studied and its function and regulation remain to be completely established. Moreover, MRP3 is known to transport various bile salts and clinical drugs [41-44]. In this study, our results revealed a positive correlation between MRP3 and PXR expression $(P=0.0023)$, and in three independent cohort, we found increased MRP3 expression in samples expressing high levels of exogenous and endogenous PXR. As PXR is a transcription factor, we used luciferase reporter assays to assess whether PXR binds to the MRP3 promoter to activate its transcription. Our results revealed that PXR can directly bind to the MRP3 promoter to activate its transcription. To determine the precise PXR-binding site within the MRP3 promoter, we constructed luciferase constructs containing different regions of the MRP3 promoter. Luciferase reporter and ChIP assays determined that the PXR-binding sites are located between -884 to -620bp of the MRP3 promoter. Further studies reveal that the key binding sites are "-_-_-_-AGGGA-" (-787 to -783bp) between -884 to $-620 \mathrm{bp}$ of the MRP3 promoter. These data showed that PXR activates MRP3 transcription by directly binding to its promoter, which increases the drug efflux capacity and results in resistance to platinum drugs. Unfortunately, the crucial sites within the PXR to bind MRP3 promoter are still unknown, and further studies employing site-directed mutagenesis of putative binding sites in PXR are required to clarify this issue. In addition, we also tested other known PXR target genes, and found that the MRP5, MDR1 and OATP-A expression were slightly up-regulated in the PXR over- expression cells (Additional file 1: Figure S1). Although the other target genes also appeared increasing, we still think that PXR increases the drug efflux capacity at least in part by directly activating MRP3 transcription according to our data of function, mechanism and restoring experiments.

To further determine the importance of PXR in CRC, a correlation analysis between PXR expression and the OS of patients was performed. Kaplan-Meier and Coxregression analysis revealed significantly reduced OS of patients with high PXR expression. Further studies demonstrate that PXR is an unfavorable and independent prognostic factor for male or clinical stage I + II CRC patients. These results indicate that PXR is a potential biomarker for predicting outcome in CRC patients.

\section{Conclusion}

In conclusion, PXR enhances the resistance of tumor cells to the chemotherapeutic agent L-OHP via direct transcriptional activating MRP3 expression, increasing the LOHP efflux capacity of CRC cells (Fig. 8). However, whether this mechanism is generally applicable to all chemotherapeutic drugs, to only specific platinum-based drugs, or only to L-OHP requires further investigation.

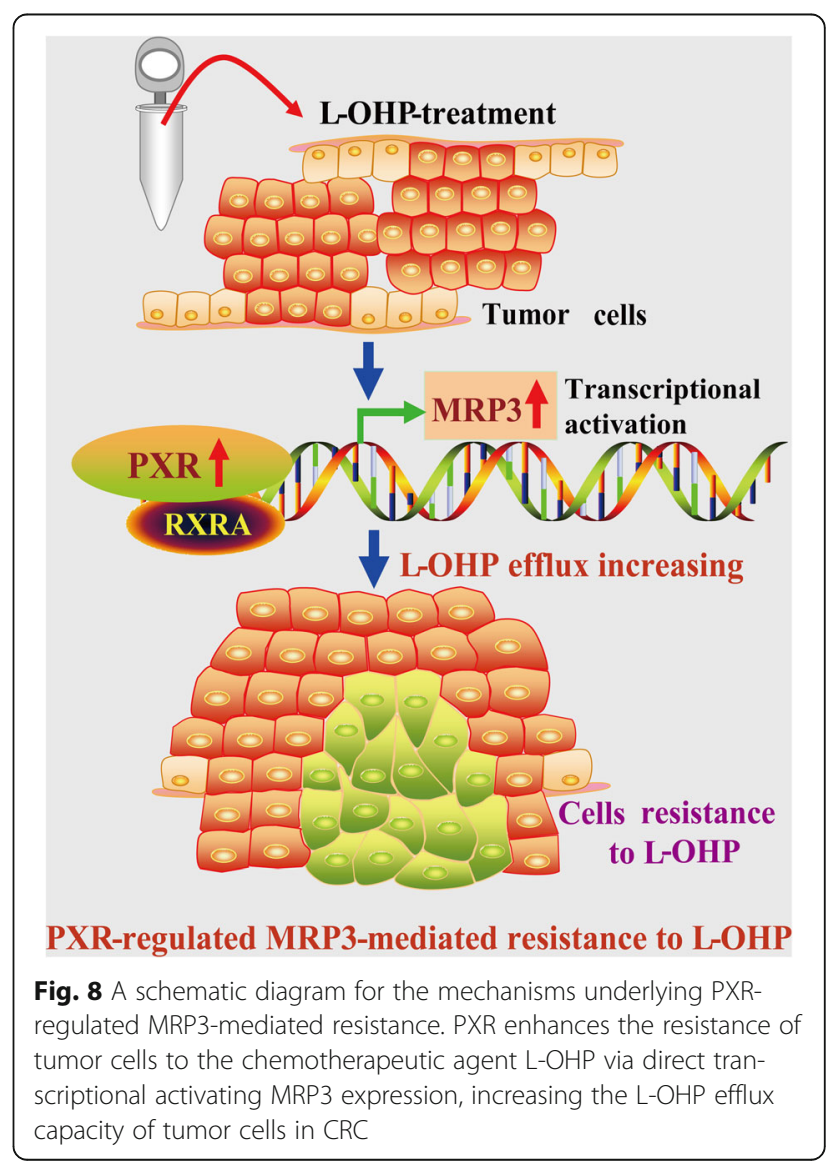




\section{Additional files}

Additional file 1: The primers, clone number, correlation of expression with clinicopathological features, prognostic factors and expression of PXR targets in this study. (DOC $289 \mathrm{~kb}$ )

Additional file 2: Database of PXR $I H C$ and clinical characteristics of colorectal cancer patients. (XLSX $20 \mathrm{~kb}$ )

\section{Abbreviations}

ChIP: Chromatin-immunoprecipitation; CRC: Colorectal cancer; IHC: Immunohistochemical; L-OHP: Oxaliplatin; MRP3: Multidrug resistancerelated protein 3; OS: Overall survival; PXR: Pregnane $X$ receptor; s.d.: Standard deviation; TCGA: The Cancer Genome Atlas; TMA: Tissue microarray; WB: Western blot

\section{Acknowledgements}

The authors would like to thank all the patients involved in this study.

\section{Funding}

This work was supported by the National Natural Science Foundation of China (No. 81172114).

\section{Availability of data and materials}

The datasets supporting the conclusions of this article are included within the additional files.

\section{Authors' contributions}

$J$ conceived this study. YD, ZW and GX studied the function of PXR. JL, YD and ZW evaluated the mechanisms. CL, WZ, GM, CX performed IHC, data processing and statistical analysis. YD and ZW wrote the manuscript. JL revised the manuscript and approved the final version. All the authors reviewed and approved the final manuscript.

\section{Competing interests}

The authors declare that they have no competing interests.

\section{Consent for publication}

Not applicable.

\section{Ethics approval and consent to participate}

The study was approved by the ethics committee of Southwest Hospital. Informed consent was obtained from all recruited patients.

\section{Publisher's Note}

Springer Nature remains neutral with regard to jurisdictional claims in published maps and institutional affiliations.

\section{Author details}

1 Department of Oncology, Southwest Hospital, Third Military Medical University, No. 29, Gaotanyan Street, Shapingba District, Chongqing 400038 People's Republic of China. 'Department of Pathology, Southwest Hospital, Third Military Medical University, No. 29, Gaotanyan Street, Shapingba District, Chongqing 400038, People's Republic of China.

Received: 20 November 2016 Accepted: 20 March 2017

Published online: 29 March 2017

\section{References}

1. Torre LA, Bray F, Siegel RL, Ferlay J, Lortet-Tieulent J, Jemal A. Globalcancer statistics, 2012. CA Cancer J Clin. 2015;65:87-108.

2. Kuhry E, Schwenk W, Gaupset R, Romild U, Bonjer J. Long-term outcome of laparoscopic surgery for colorectal cancer: a cochrane systematic review of randomized ontrolled trials. Cancer Treat Rev. 2008:34:98-504

3. Siegel R, DeSantis S, Jemal A. Colorectal Cancer Statistics, 2014. CA Cancer J Clin. 2014:64:104-17.

4. Van Cutsem E, Cervantes A, Nordlinger B, Arnold D, on ehalf of the ESMO GuidelinesWorking Group. Metastatic colorectal cancer: ESMO Clinical Practice Guidelines for iagnosis, treatment and follow-up. Ann Oncol. 2014;25:iii1-9.
5. Simmonds PC. Palliative chemotherapy for advanced colorectal cancer: systematic review and meta-analysis. colorectal Cancer Collaborative Group. Br Med J. 2000;321:531-5.

6. Ragnhammar P, Hafström L, Nygren P, Glimelius B. A ystematic overview of chemotherapy effects in colorectal ancer. Acta Oncol. 2001;40:282-308.

7. Chabner BA, Roberts Jr TG. Timeline: chemotherapy and the war on cancer. Nat Rev Cancer. 2005;5:65-72.

8. Tournigand C, Andre T, Achille E, Lledo G, Flesh M, Mery-Mignard D, Quinaux E, Couteau C, Buyse M, Ganem G, et al. FOLFIRI followed by FOLFOX6 or the reverse sequence in advanced colorectal cancer: a randomized GERCOR study. J Clin Oncol. 2004;22:229-37.

9. Schmoll HJ, Van Cutsem E, Stein A, Valentini V, Glimelius B, Haustermans K, Nordlinger B, van de Velde CJ, Balmana J, Regula J, et al. ESMO Consensus Guidelines for management of patients with colon and rectal cancer. A personalized approach to clinical decision making. Ann Oncol. 2012;23: 2479-516.

10. Kliewer SA, Moore JT, Wade L, Staudinger JL, Watson MA, Jones SA, McKee DD, Oliver BB, Willson TM, Zetterström RH, et al. An orphan nuclear receptor activated by pregnanes defines a novel steroid signaling pathway. Cell. 1998;92:73-82

11. Nishimura M, Naito S, Yokoi T. Tissue-specific mRNA expression profiles of human nuclear receptor subfamilies. Drug Metab Pharmacokinet. 2004;19: 135-49.

12. Pondugula SR, Mani S. Pregnane xenobiotic receptor in cancer pathogenesis and therapeutic response. Cancer Lett. 2013;328:1-9.

13. Qiao E, Ji M, Wu J, Ma R, Zhang X, He Y, Zha Q, Song X, Zhu LW, Tang J. Expression of the PXR gene in various types of cancer and drug resistance. Oncol Lett. 2013:5:1093-100.

14. Miki Y, Suzuki T, Kitada K, Yabuki N, Shibuya R, Moriya T, Ishida T, Ohuchi N, Blumberg B, Sasano H. Expression of the steroid and xenobiotic receptor and its possible target gene, organic anion transporting polypeptide-A, in human breast carcinoma. Cancer Res. 2006:66:535-42.

15. Yue $X$, Akahira J, Utsunomiya H, Miki Y, Takahashi N, Niikura H, Ito K, Sasano H, Okamura K, Yaegashi N. Steroid and xenobiotic receptor (SXR) as a possible prognostic marker in epithelial ovarian cancer. Pathol Int. 2010;60:400-6.

16. Jiang H, Chen K, He J, Pan F, Li J, Chen J, Chen W, Liang H. Association of pregnane $\mathrm{X}$ receptor with multidrug resistancerelated protein 3 and its role in human colon cancer chemoresistance. J Gastrointest Surg. 2009;13:1831-8.

17. Masuyama H, Hiramatsu Y, Kodama J, Kudo T. Expression and potential roles of pregnane $X$ receptor in endometrial cancer. J Clin Endocrinol Metab. 2003;88:4446-54.

18. Chen $Y$, Nie D. Pregnane $X$ receptor and its potential role in drug resistance in cancer treatment. Recent Pat Anticancer Drug Discov. 2009;4:19-27.

19. Chen Y, Tang Y, Guo C, Wang J, Boral D, Nie D. Nuclear receptors in the multidrug resistance through the regulation of drug-metabolizing enzymes and drug transporters. Biochem Pharmacol. 2012:83:1112-26.

20. Chen T. Overcoming drug resistance by regulating nuclear receptors. Adv Drug Deliv Rev. 2010;62:1257-64.

21. Harmsen S, Meijerman I, Beijnen JH, Schellens JH. The role of nuclear receptors in pharmacokinetic drug-drug interactions in oncology. Cancer Treat Rev. 2007;33:369-80.

22. Meijerman I, Beijnen $\mathrm{JH}$, Schellens $\mathrm{JH}$. Herb-drug interactions in oncology: focus on mechanisms of induction. Oncologist. 2006;11:742-52.

23. Willson TM, Kliewer SA. PXR, CAR and drug metabolism. Nat Rev Drug Discov. 2002;1:259-66.

24. Gao Q, Qiu SJ, Fan J, Zhou J, Wang XY, Xiao YS, Xu Y, Li YW, Tang ZY. Intratumoral balance of regulatory and cytotoxic $T$ cells is associated with prognosis of hepatocellular carcinoma after resection. J Clin Oncol. 2007;25: 2586-93.

25. Han F, Liu W, Xiao H, Dong Y, Sun L, Mao C, Yin L, Jiang X, Ao L, Cui Z, et al. High expression of SOX30 is associated with favorable survival in human lung adenocarcinoma. Sci Rep. 2015;5:13630.

26. Han F, Liu W, Jiang X, Shi X, Yin L, Ao L, Cui Z, Li Y, Huang C, Cao J, et al. SOX30, a novel epigenetic silenced tumor suppressor, promotes tumor cell apoptosis by transcriptional activating p53 in lung cancer. Oncogene. 2015: 34:4391-402.

27. Bendall SC, Simonds EF, Qiu P, Amir e-AD, Krutzik PO, Finck R, Bruggner RV Melamed R, Trejo A, Ornatsky Ol, et al. Single-cell mass cytometry of differential immune and drug responses across a human hematopoietic continuum. Science. 2011;332:687-96. 
28. Chang Q, Ornatsky Ol, Koch CJ, Chaudary N, Marie-Egyptienne DT, Hill RP, Tanner SD, Hedley DW. Single-cell measurement of the uptake, intratumoral distribution and cell cycle effects of cisplatin using mass cytometry. Int J Cancer. 2015;136:1202-9.

29. Fienberg HG, Simonds EF, Fantl WJ, Nolan GP, Bodenmiller B. A platinumbased covalent viability reagent for single-cell mass cytometry. Cytometry A. 2012:81:467-75

30. Zi X, Guo Y, Simoneau AR, Hope C, Xie J, Holcombe RF, Hoang BH. Expression of Frzb/secreted Frizzled-related protein 3, a secreted Wnt antagonist, in human androgen-independent prostate cancer PC-3 cells suppresses tumor growth and cellular invasiveness. Cancer Res. 2005;65: 9762-70.

31. Campa D, Butterbach K, Slager SL, Skibola CF, de Sanjosé S, Benavente Y Becker N, Foretova L, Maynadie M, Cocco P, et al. A comprehensive study of polymorphisms in the $A B C B 1, A B C C 2, A B C G 2, N R 112$ genes and lymphoma risk. Int J Cancer. 2012;131:803-12.

32. Xie W, Yeuh MF, Radominska-Pandya A, Saini SP, Negishi Y, Bottroff BS, Cabrera GY, Tukey RH, Evans RM. Control of steroid, heme, and carcinogen metabolism by nuclear pregnane X. receptor and constitutive androstane receptor. Proc Natl Acad Sci U S A. 2003;100:4150-5.

33. Gupta D, Venkatesh M, Wang H, Kim S, Sinz M, Goldberg GL, Whitney K, Longley C, Mani S. Expanding the roles for pregnane $X$ receptor in cancer: proliferation and drug resistance in ovarian cancer. Clin Cancer Res. 2008;14: 5332-40.

34. Basseville A, Preisser L, de Carné TS, Boisdron-Celle M, Gamelin E, Coqueret $\mathrm{O}$, Morel A. Irinotecan induces steroid and xenobiotic receptor (SXR) signaling to detoxification pathway in colon cancer cells. Mol Cancer. 2011;10:80

35. Harmsen S, Meijerman I, Febus CL, Maas-Bakker RF, Beijnen JH, Schellens JH. PXR-mediated induction of P-glycoprotein by anticancer drugs in a human colon adenocarcinoma-derived cell line. Cancer Chemother Pharmacol. 2010;66:765-71.

36. Chen $Y$, Tang $Y$, Chen $S$, Nie D. Regulation of drug resistance by human pregnane X receptor in breast cancer. Cancer Biol Ther. 2009;8:1265-72.

37. Chen $Y$, Tang $Y$, Wang MT, Zeng S, Nie D. Human pregnane $X$ receptor and resistance to chemotherapy in prostate cancer. Cancer Res. 2007;67:10361-7.

38. Synold TW, Dussault I, Forman BM. The orphan nuclear receptor SXR coordinately regulates drug metabolism and efflux. Nat Med. 2001;7:584-90.

39. Mensah-Osman E, Lin H-L, Reinke D, Hollenberg P, Baker L. Ecteinascidin743 is a potent inhibitor of P450 3A4 enzyme and accumulates cytoplasmic PXR to inhibit transcription of P450 3A4 and MDR1: implications for the enhancement of cytotoxicity to chemotherapeutic agents in osteosarcoma. J Clin Oncol (Meeting Abstracts). 2005;23:9026.

40. Tolson AH, Wang H. Regulation of drug-metabolizing enzymes by xenobiotic receptors: PXR and CAR. Adv Drug Deliv Rev. 2010;62:1238-49.

41. Hirohashi T, Suzuki H, Takikawa H, Sugiyama Y. ATP-dependent transport of bile salts by rat multidrug resistance associated protein 3 (Mrp3). J Biol Chem. 2000;275:2905-10.

42. Lagas JS, Fan L, Wagenaar E, Vlaming ML, van Tellingen $\mathrm{O}$, Beijnen $\mathrm{JH}$, Schinkel AH. P-glycoprotein (P-gp/Abcb1), Abcc2, and Abcc3 determine the pharmacokinetics of etoposide. Clin Cancer Res. 2010;16:130-40.

43. Kool M, van der Linden M, de Haas M, Scheffer GL, de Vree JM, Smith AJ, Jansen G, Peters GJ, Ponne N, Scheper RJ, et al. MRP3, an organic anion transporter able to transport anti-cancer drugs. Proc Natl Acad Sci U S A. 1999;96:6914-9.

44. Zelcer N, Saeki T, Reid G, Beijnen JH, Borst P. Characterization of drug transport by the human multidrug resistance protein 3 (ABCC3). J Biol Chem. 2001;276:46400-7.

\section{Submit your next manuscript to BioMed Central and we will help you at every step:}

- We accept pre-submission inquiries

- Our selector tool helps you to find the most relevant journal

- We provide round the clock customer support

- Convenient online submission

- Thorough peer review

- Inclusion in PubMed and all major indexing services

- Maximum visibility for your research

Submit your manuscript at www.biomedcentral.com/submit

) Biomed Central 